BULL. AUSTRAL. MATH, SOC.

VOL. 30 (1984), 271-287

\title{
THE MATHEMATICS OF HYSTERESIS
}

\author{
ULRICH HORNUNG
}

A survey over three different approaches to model hysteresis phenomena is given. The starting point for mathematical models are
(a) memory,
(b) population, or
(c) spatial distribution.

In any case the hysteresis effect is interpreted using an averaging procedure over the inner state of the system in consideration.

\section{Introduction}

Hysteresis can be observed in many different fields of science. It is well known that for a ferromagnetic material the magnetic induction $B$ is not a single valued function of the magnetic field $H$. If $H$ is a periodic function of time $t$, then the variation of $B$ lags behind that of $H$, and we obtain a loop of the type shown in Figure l. If a different input signal $t \rightarrow H(t)$ is used a different output signal $t \rightarrow B(t)$ is observed, and in general also a different graph in the $H-B$-plane.

Similar phenomena are encountered in ferroelectric materials, where the variables in question are the electric field and the polarization, and in elasticity. In the latter case the variables for which hysteresis

Received 9 April 1984. This work was supported by the Deutsche Forschungsgemeinschaft (Grant 477/692/83).

Copyright Clearance Centre, Inc. Serial-fee code: 0004-9727/84 $\$ 2.00+0.00$. 


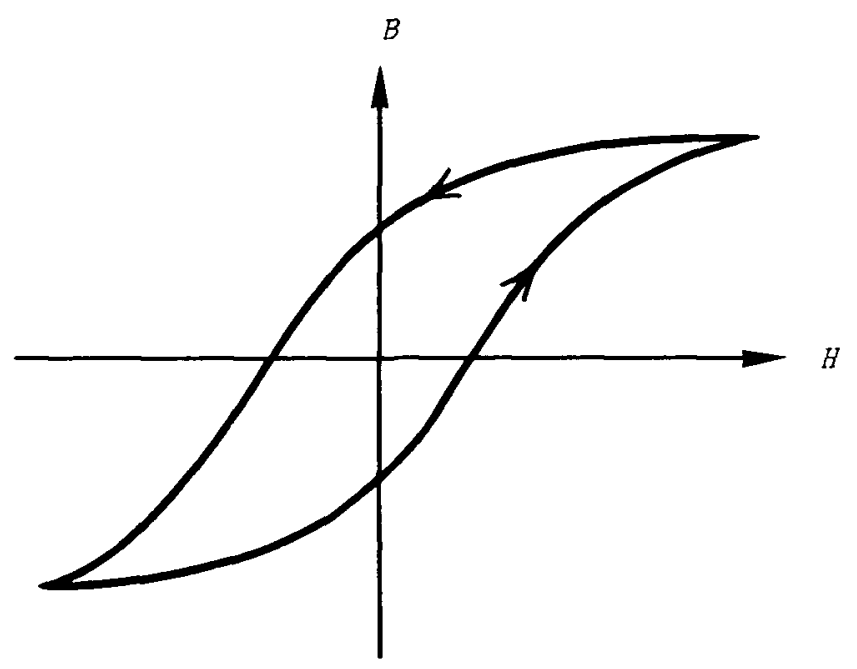

FIGURE 1

occurs are stress and strain. For all these examples the area enclosed by the loop corresponds to the energy which is lost as heat if a full cycle is performed.

Another example is a crowd of moths which fly towards two lights of variable intensity. If we consider the relation between the intensities and the fraction of moths being attracted by one of the lights we observe some type of hysteresis. A similar example is provided by two political parties and their voters.

Finally we mention the example of unsaturated water flow through porous media. Here the two variables for which hysteresis has been observed are the water content and the capillary pressure head.

Though hysteresis phenomena have been known for about one hundred years (cf. Rayleigh [25], Ewing [17]), a rigorous mathematical treatment has been tried only very recently. The purpose of a model is not only to make it accessible to mathematical theory and so to contribute to a better understanding of the phenomenon. A model is also a means to be used for computer simulations of processes in which hysteresis is an essential feature.

Before dealing with various theoretical approaches we give a 
preliminary definition. We say that the relation of an input signal $v$ and an output signal $u$ is of hysteresis type if all possible points $(v, u)$ form a set $M$ in the phase plane $v-u$ surrounded by some outer loop such that:

(A) each point within the loop is attainable, that is $M$ is equal to the interior of the loop;

(B) the forthcoming behaviour of the system cannot be predicted from the knowledge of the actual point in the set $M$ alone;

(C) the scanning curves in the set $M$ depend on the frequency of the input signal, that is, on the derivative of $v$ with respect to time.

The properties $(A),(B)$, and (C) can be found in many systems with hysteresis. It is clear that not all properties need be shared by all systems. Some authors neglect some of them. There is a school saying that hysteresis is a phenomenon in which (c) is not true, that is, in which the curves in the phase plane remain unchanged if the time scale is changed. It is our opinion that a general model should be compatible with observations from all experiments.

\section{Memory models}

\subsection{ORDINARY DIFFERENTIAL EQUATIONS}

The first attempt to study mathematical models for hysteresis was made by Duhem [2]-[10] in a series of papers. He studied hysteresis in the context of physical chemistry and investigated the role of the inequality of clausius in thermodynamics. A result of his work was that within a hysteresis loop there are two families of curves, the family of ascending curves and the family of descending curves. Through every point $(v, u)$ passes exactly one curve of each family. Those points where these two curves are parallel were called "natural states" of the system.

A mathematical model of this approach can be given as an ordinary differential equation

$$
\frac{d}{d t} u(t)=f\left(u(t), v(t), \operatorname{sign}\left(\frac{d}{d t} v(t)\right)\right) \cdot \frac{d}{d t} v(t) .
$$

The two families of curves are obtained from this equation as the solutions 
of the two differential equations

$$
\frac{d u}{d v}=f(u, v,+1)
$$

and

$$
\frac{d u}{d v}=f(u, v,-1)
$$

It is obvious that properties (B) and (C) are not satisfied by hysteresis systems of this kind.

\subsection{MEMORY OPERATORS}

A phenomenological study of hysteresis leads to the idea that it is caused by some time lag or a memory of the system. Therefore it seems to be natural to use a mathematical model that relates the value of the output signal $u(t)$ to the history $v(\tilde{t}), \tilde{t} \leq t$, of the input signal. Hence we have an equation of the form

$$
u(t)=H(v(\cdot), t, u(0)) .
$$

where $v(\cdot) \in C^{0}[0, t]$ is the history of $v$ and $i \mu(0)$ is the initial value of $u$. The operator $H$ describes the dynamic behaviour of the system in consideration. In some special cases this operator may have the form of a convolution integral

$$
H\left(v(\cdot), t, u_{0}\right)=\int_{0}^{t} K(t-s, t) G(v(s)) d s+u_{0} .
$$

This approach was first used by Krasnosel'skii [20]. It was studied systematically and in connection with partial differential equations by visintin [26]. It is clear that very general systems can be modelled using (2.3), but the physical interpretation of the operator $H$ is not evident.

\section{Population models}

\subsection{THEORY OF INDEPENDENT DOMAINS}

Ewing [17] was one of the first who explained magnetic phenomena in terms of forces between atoms. Weiss [28] studied magnetic fields of molecules. Later Weiss and de Freudenreich [29] developed the idea of elementary magnetic domains further. This leads to the idea of independent domains described by Preisach [24], which was put into a more abstract form 
by Néel [22], [23]. Later this was rediscovered independently by Everett [13]-[16] and Enderby [11], [12].

The basic assumption of the theory is that the system under consideration is a population of domains each of which reacts in a very elementary way to the input signal $v$. Each domain behaves like a switch, a typical example for which is a thermostat. The switch has only two possible states, say 0 and 1 ; it switches from 0 to $l$ if the input $v$ becomes larger than an upper threshold $x$, it switches from 1 to 0 if the input becomes smaller than a lower threshold $y$; see Figure 2 .

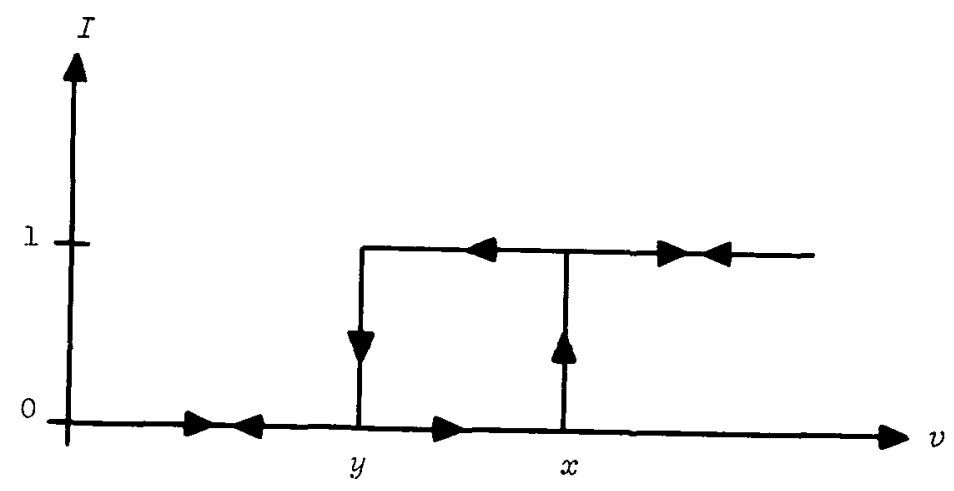

FIGURE 2

The hysteresis loop of a switch can be modelled by the evolution equation

$$
\partial_{t} I(t, x, y) \in-A(I(t, x, y), v(t), x, y) .
$$

Here $A(\cdot, v, x, y)$ is the set valued operator from $\mathbb{R}$ to $\mathbb{R}$ given by the graph $\tilde{A}(\cdot, v, x, y)=\{(a, b): b \in A(a, v, x, y)\}$ in $\mathbf{R} \times \mathbf{R}$,

$$
\tilde{A}(\cdot, v, x, y)= \begin{cases}(\{0\} \times \mathbf{R}), v<y, \\ (\{0\} \times(-\infty, 0]) \cup([0,1] \times\{0\}) \cup(\{1\} \times[0,+\infty)\}, \\ \{0\} \times \mathbf{R}, x<v ; & y \leq x,\end{cases}
$$


cf. Brezis [1]. It is easy to see that for all $v, x$, and $y$ the operator $\tilde{A}(\cdot, v, x, y)$ is maximal monotone; that is, we have

$$
\left(a_{1}, b_{1}\right),\left(a_{2}, b_{2}\right) \in \tilde{A} \Rightarrow\left(a_{1}-a_{2}\right) \cdot\left(b_{1}-b_{2}\right) \geq 0
$$

and $\tilde{A}$ is maximal in $\mathbf{R} \times \mathbf{R}$ of this type.

A switch of the type described above is a very simple example of a system with hysteresis. Obviously properties $(A),(B)$, and (C) do not apply to the relation between $v$ and $I$.

A general system considered by the theory of independent domains is a population of switches each of which reacts independently of the others to the input signal. The state $u$ of the whole system is a weighted average of the states of all domains

$$
u(t)=\int I(t, x, y) d F(x, y)
$$

Here $F$ is a two-dimensional distribution function of a measure

$$
\mu(M)=\int_{M} d F(x, y)
$$

with support in the half plane $\{(x, y): y \leq x\}$. It is understood that $F$ can be defined by $\mu$ via

$$
F(x, y)=\mu(\{(\tilde{x}, \tilde{y}): \tilde{x} \leq x, \tilde{y} \leq y\}) .
$$

Evidently $\mu(M)$ is the fraction of domains in the population having thresholds $(x, y) \in M$.

Since $I(t, \cdot, \cdot)$ is the characteristic function of the set

$$
M(t)=\{(x, y): I(t, x, y)=1\},
$$

the state of the system is

$$
u(t)=\mu(M(t))=\int_{M(t)} d F(x, y)=\int I(t, x, y) d F(x, y) .
$$

Therefore in general the inner state of the system is not known if only $\{v(t), u(t)\}$ is given, but it is described by $I(t, \cdot, \cdot)$ or by $M(t)$. This inner state has been called the "domain complexion". It is easy to see that if $M(t)$ has been generated by some signal $v(\tilde{t}), \tilde{t} \leq t$, then it, can be represented as a union 


$$
M(t)=\bigcup_{j \in J}\left(-\infty, x_{j}\right) \times\left(-\infty, y_{j}\right)
$$

where the $x_{j}$ and $y_{j}$ are relative maxima and minima that occurred in the history of $v$.

According to the theory of independent domains, hysteresis systems are described by equations (3.1) and (3.2). Obviously properties (A) and (B) apply to general systems of this kind, but property (C) does not. The behaviour of a system can be predicted if the measure $\mu$ or equivalently the distribution function $F$ is known. For instance the primary scanning curves, that is, the ascending and the descending boundaries of the hysteresis loop, can be obtained very easily from $F$. These curves are the maximal monotone extensions of the sets

$$
\{(x, y): y=F(x, x)\} \text { and }\{(x, y): y=F(\infty, x)\} .
$$

Besides the most elementary systems, namely switches, for which the support of the measure $\mu$ is a singleton, there is an interesting class with relatively simple behaviour. If the support of $\mu$ is a monotone set in $\mathbb{R}^{2}$, then all scanning curves within the hysteresis loop are horizontal. In this case property (B) does not hold, while (A) may or may not hold depending on the smoothness of the measure.

The class of systems described previously is one for which the structure is known once the two primary scanning curves are given. Another class of this type are those systems for which the similarity hypothesis

$$
F(x, y)=G(x) \cdot H(y)
$$

is satisfied. It is clear that if now

$$
x \rightarrow G(x) \cdot H(x) \text { and } x \rightarrow G(\infty) \cdot H(x)
$$

are measured (see (3.3)) then $G$ and $H$, and so $F$, are determined. Systems of this tvpe have been studied by Mualem [21] and Haverkamp and Parlange [18] for seepage flow problems. In a general situation a twodimensional distribution has to be determined, a problem for which there is no simple procedure.

An application of the theory of independent domains to parabolic problems was investigated by Visintin [27]. 


\subsection{REVERSIBLE PROCESSES}

In his paper on hysteresis Preisach [24] mentioned systems consisting of many pairs of electric condensators and resistances. Each of the pairs, which we again call domains, is influenced by some voltage $v$ : see Figure 3.
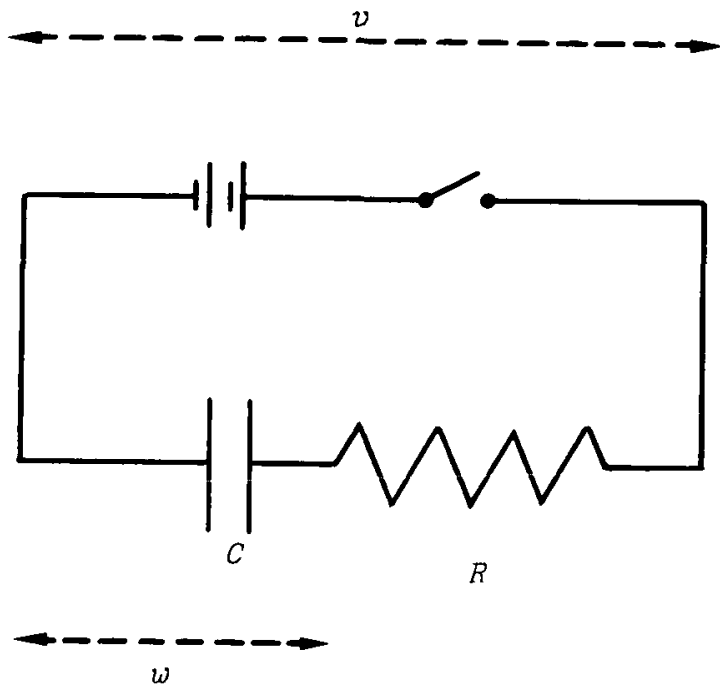

FIGURE 3

In this case the voltage $w$ at the condensator obeys the law

$$
\dot{w}=-\frac{\jmath}{R C}(w-v)
$$

If we denote the time constant by

$$
z=\frac{1}{R C} \text {, }
$$

we have

$$
\partial_{t} w(t, z)=-z \cdot(w(t, z)-v(t))
$$

indicating that different domains may have different time constants. The state of the whole system under consideration is assumed to be a weighted average of the states of all domains; that is,

$$
u(t)=\int w(t, z) d F(z)
$$


In this context $F$ is the distribution function of a discrete or continuous measure $\mu$, where $\mu(M)$ describes the fraction of domains in the system having time constants $z \in M$.

Since each domain has linear behaviour, the whole system behaves linearly. By this we mean that the transition operator $v \rightarrow u$ is linear. Therefore the system is fully understood if the response $u$ to a $\delta$-impuise is known. In the situation described above the unit response is

$$
u(t)=\int z e^{-z t} d F(z) .
$$

Alternatively linear systems can also be described by their impedance

$$
\phi(w)=u(t) / v(t)
$$

for periodic signals $v(t)=e^{i \omega t}$. Here we have

$$
\phi(\omega)=\int \frac{d F(z)}{1+(i \omega / z)} .
$$

Processes of this type are reversible in the sense that for extremely slow inputs the $v$-u-relation is almost a line in the phase plane. Since it is reasonable that the measure $\mu$ has compact support in the half line of positive numbers, we have, for very small frequences $\omega$,

$$
\phi(\omega) \approx \int d F(z)
$$

and therefore

$$
u(t) \approx v(t) \int d F(z)
$$

A natural generalization of systems described here are populations of domains each of which can be modelled by an evolution equation

$$
\partial_{t} w(t, z)=-A(w(t, z), v(t), z) \text {. }
$$

Here $A(\cdot, v, z)$ is a nonlinear monotone operator. As before we assume that the state of the system to be described is given by an integral

$$
u(t)=\int w(t, z) d F(z) .
$$

\subsection{GENERAL POPULATIONS}

It is evident that the systems studied in paragraphs 3.1 and 3.2 have 
the same general mathematical model, namely equations (3.7) and (3.8). In any case we think of populations of domains described by parameters $(x, y)$ or $z$, respectively. The dynamics of each domain is given by (3.7), where we assume that $A(\cdot, v, z)$ is a maximal monotone operator. Both reversible and irreversible processes can be modelled in this framework, depending on whether or not $A$ is strictly monotone. An application of this idea to ferromagnetism has been published by Hornung [19].

\section{Spatial models}

The assumption of the theory of independent domains is that the system is a population of domains which do not interact. In this paragraph we study systems which are distributed in space such that there is an influence from each point to its neighbours. In this case it is natural to assume that the state of the whole system is an average with respect to the space variable. Let $\Omega$ be a bounded domain in $R^{n}$ and the output variable $u$ be given by

$$
u(t)=f_{\Omega} w(t, x) d x=\int_{\Omega} w(t, x) d x / \int_{\Omega} d x .
$$

Now $w$ denotes the inner state of the system which may be influenced by the input variable $v$ in different ways.

The first example is obtained from linear relations of the type described in equation (3.5) by introducing a diffusion process that takes place in space. In this case we have

(4.2a) ${ }_{2} w(t, x)=\cdot b \Delta w(t, x)-a(x) \cdot(w(t, x)-v(t)), x \in \Omega$,

where $b$ denotes the diffusivity. If we impose boundary conditions on $\partial \Omega$, for example homogeneous Dirichlet conditions

$$
w(t, x)=0, x \in \partial \Omega,
$$

we obtain a linear transition operator. It is easy to calculate the impedance. If $\varphi$ is the solution of the elliptic problem

$$
\left\{\begin{array}{l}
-b \Delta \varphi(x)+(a(x)+i \omega) \varphi(x)=a(x), x \in \Omega, \\
\varphi(x)=0, x \in \partial \Omega,
\end{array}\right.
$$

the impedance is given by 


$$
\phi(\omega)=f_{\Omega} \varphi(x) d x .
$$

If $a(x)$ is constant and $\Omega$ is the unit ball in $R^{n}$, problem (4.3) can be solved explicitly. Using

$$
\lambda=1 /\left(1+\frac{i \omega}{a}\right), \mu^{2}=-(a+i \omega) / b \text {, and } r=|x| \text {, }
$$

we have

$$
\varphi(x)=\lambda \cdot(1-\psi(\mu r) / \psi(\mu)),
$$

where $\psi(s)$ is a bounded solution of

$$
\psi^{\prime \prime}+\frac{n-1}{s} \psi^{\prime}+\psi=0, \quad,=\frac{d}{d s} .
$$

It is well known that we have

$$
\psi(s)= \begin{cases}\cos s, & n=1, \\ J_{0}(s), & n=2, \\ j_{0}(s), & n=3,\end{cases}
$$

where $J_{0}$ and $j_{0}$ are the cylindrical and spherical Bessel functions of order zero respectively. Now simple integration gives the impedance

$$
\phi(\omega)=\lambda \cdot\left(1-\frac{1}{\mu} \psi(\mu)\right),
$$

where the value $\psi$ is given by

$$
\Psi(\mu)= \begin{cases}\tan \mu, n=1, & \\ 2 J_{1}(\mu) / J_{0}(\mu), & n=2, \\ 3((1 / \mu)-\cot \mu), & n=3 .\end{cases}
$$

It is clear that for $b \rightarrow 0$ we have $\phi(\omega) \rightarrow \lambda$, the limit case of vanishing diffusivity.

In the second example we consider a diffusion process where now the input $v$ enters as the boundary values. Adopting linear equations we have

$$
\left\{\begin{array}{l}
\partial_{t} w(t, x)=b \Delta w(t, x), x \in \Omega, \\
w(t, x)=v(t), \quad x \in \partial \Omega .
\end{array}\right.
$$


In this case the impedance is obtained by solving the elliptic problem

$$
\left\{\begin{array}{l}
b \Delta \varphi(x)-i \omega \varphi(x)=0, x \in \Omega, \\
\varphi(x)=I, x \in \partial \Omega,
\end{array}\right.
$$

from which the impedance

$$
\Phi(\omega)=f_{\Omega} \varphi(x) d x
$$

is calculated. If we introduce $\mu^{2}=-i \omega / b$, then using the same functions $\psi$ and $\Psi$ as before we have

$$
\varphi(x)=\psi(\mu r) / \psi(\mu)
$$

and

$$
\Phi(\omega)=\frac{1}{\mu} \Psi(\mu)
$$

Since the examples (4.2) and (4.4) describe linear processes there is no bounded hysteresis loop in the $v$-u-plane for these systems. It is clear that there are many ways to introduce nonlinearities which may lead to better models for hysteresis. The following example is of special interest in hydrology.

Unsteady water flow through porous media is governed by the differential equation

$$
\partial_{t} w(t, x)=\operatorname{div}(K(w(t, x)) \operatorname{grad} p(t, x)),
$$

where $w$ is the water content, $p$ the capillary potential, and $K$ the hydraulic conductivity. Generally it is assumed that there is a well defined nonlinear relation between $p$ and $w$; see Figure 4. This functions and the function $K(\cdot)$ depend on the microscopic structure of the medium.

To measure the function $p \rightarrow w$ in a laboratory amounts to making an experiment of the following type. At one side of a probe of the medium a certain value $v$ of the variable $p$ is applied, while the other sides are sealed using some impermeable material. In this way we impose boundary conditions on the boundary $\partial \Omega=\Gamma_{0}+\Gamma_{1}$ of the domain $\Omega$; see Figure 5 . 


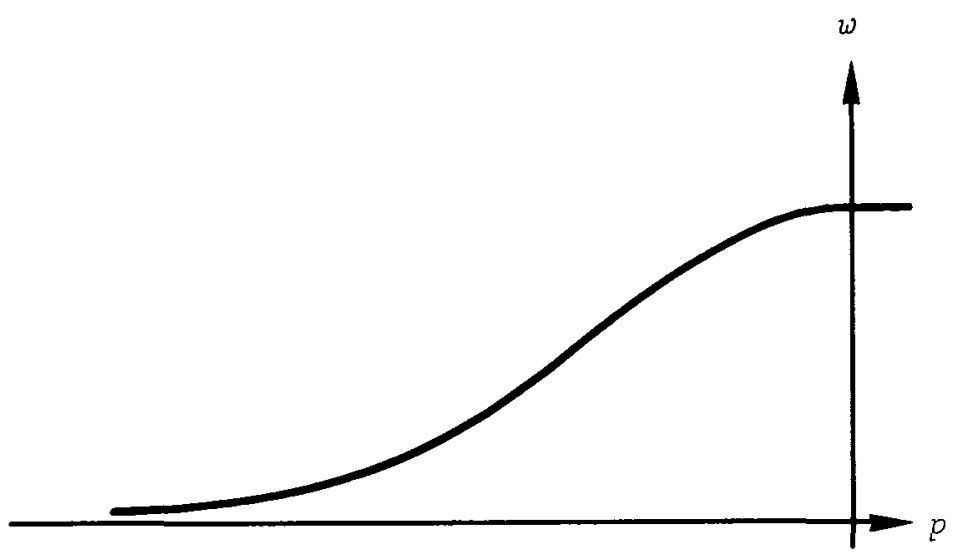

FIGURE 4

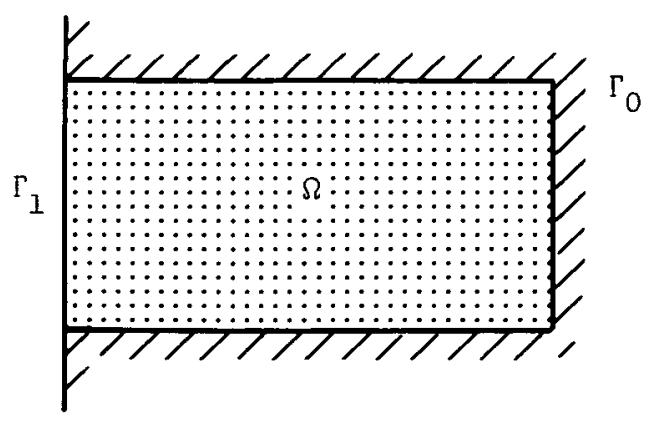

FIGURE 5 
$(4.6 \mathrm{~b})$

$$
\left\{\begin{array}{l}
\partial_{v} p(t, x)=0, x \in \Gamma_{0}, \\
p(t, x)=v, x \in \Gamma_{1} .
\end{array}\right.
$$

For $t \rightarrow \infty$ the solution $p$ of problem (4.6) becomes constant in $\Omega$, namely $p=v$, no matter which initial function $p(0, x)$ was used. Therefore the water content $w$ becomes constant in $\Omega$, and one pair of the function $p \rightarrow w$ is determined.

In practice the process described by (4.6) is not observed for arbitrarily large $t$, but the average water content (4.1) is measured at finite time. At this time the distributions of $p$ and $w$ may not yet be constant in $\Omega$. Therefore the measurement gives the transition operator $v \rightarrow u$ rather than the function $p \rightarrow w$. It is clear that the result depends strongly on the size of the probe and on the speed at which the experiment is performed.

The situation becomes more complicated if we consider inhomogeneous media. If the conductivity $K$ varies in space over several orders of magnitude then the rate at which an equilibrium is reached within the domain $\Omega$ varies at the same rate. Therefore the experiment may be far from a steady state, and hence the $v$-u-relation may be of hysteresis type.

A mathematical study of this phenomenon for highly oscillating conductivities is presently in consideration and will be published elsewhere.

\section{Conclusion}

The three types of mathematical models for hysteresis deal with memory, populations and systems distributed in space. In each case the main aspect is that there is an "inner state" of the system, which is the history, the state of each individual of the population, or a spatial distribution, respectively. The state of the whole system is always some mean value obtained by averaging.

It need not be emphasized that in a hysteresis phenomenon taken from the real world the three mechanisms described above may work together.

REMARK. Very recently the book M.A. Krasnosel'ski i and A.W. Pokrowskii, Systems with hysteresis, Nauka, Moscow (1983), appeared in 
Russian.

\section{References}

[1] H. Brezis, Opérateurs maximaux monotones et semi-groupes de contractions dans les espaces de Hilbert (North-Holland, Ansterdam, 1973).

[2] P. Duhem, "Die dauernden Änderungen und die Thermodynamik I: Die dauernden Ănderungen der Systeme, welche von einer einzigen normalen Ver̈̈nderlichen abhängen", Z. Phys. Chemie 22 (1897), 543-589.

[3] P. Duhem, "Die dauernden Ănderungen und die Thermodynamik II: Die Umwandlungen des Schwefels", Z. Phys. Chemie 23 (1897), 193-266.

[4] P. Duhem, "Die dauernden Ãnderungen und die Thermodynamik III:

Allgemeine Theorie der dauernden Änderungen", 2. Phys. Chemie 23 (1897), 497-541.

[5] P. Duhem, "Die dauernden Ǎnderungen und die Thermodynamik IV: Über einige Eigenschaften der Systeme, welche von einer einzigen normalen Variablen abhängen, besonders aber die Zerreissung der elastischen Körper", Z. Phys. Chemie 28 (1899), 577-618.

[6] P. Duhem, "Die dauernden Ānderungen und die Thermodynamik V: Untersuchung der Systeme, welche von zwei Veränderlichen abhängen, von denen eine keine Hysterese besitzt", Z. Phys. Cherie 33 (1900), 641-697.

[7] P. Duhem, "Die dauernden Anderungen und die Thermodvnamik VI: Die säkularen Ănderungen der Härtung", 2. Phys. Chemie 34 (1900), 312-377.

[8] P. Duhem, "Die dauernden Änderungen und die Thermodynamik VII: Über einige Anderungsmethoden, nach welchen man ein System untersuchen kann, welches von zwei Variablen mit Hysteresis abhängt", 2. Phys. Chemie 34 (1900), 683-700.

[9] P. Duhem, "Die dauernden Anderungen und die Thermodynamik VIII: Die Ungleichung von $\mathrm{Cl}$ ausius und die Hysterese", 2. Phys. Chemie 37 (1901), 91-99. 
[10] P. Duhem, "Die dauernden Ănderungen und die Thermodynamik IX: Die Hysteresis und die umkehrbaren Ănderungen", Z. Phys. Chemie (1903), 695-700.

[11] J.A. Enderby, "The domain model of hysteresis. Part I: Independent domains", Trans. Faraday Soc. 51 (1955), 835-848.

[12] J.A. Enderby, "The domain model of hysteresis. Part 2: Interacting domains", Trans. Faraday Soc. 52 (1956). 106-120.

[13] D.H. Everett and W.I. Whitton, "A general approach to hysteresis", Trans. Faraday Soc. 48 (1952), 749-757.

[14] D.H. Everett and F.W. Smith, "A general approach to hysteresis. Part 2: Development of the domain theory", Trans. Faraday Soc. 50 (1954) , 187-197.

[15] D.H. Everett, "A general approach to hysteresis. Part 3: A formal treatment of the independent domain models of hysteresis", Trans. Faraday Soc. 50 (1954), 1077-1096.

[16] D.H. Everett, "A general approach to hysteresis. Part 4: An alternative formulation of the domain model", Trans. Faraday Soc. 51 (1955), 1551-1557.

[17] J.A. Ewing, "On time-lag in the magnetication of iron", Proc. Roy. Soc. London 46 (1889), 269-286.

[18] R. Haverkamp and J.Y. Parlange, "Predicting water retention curves from particle-size distributions", Soil Sci. (to appear).

[19] U. Hornung, "Mathematical models for hysteresis effects", 2. Angew. Math. Mech. 63 (1983), T324-T325.

[20] M.A. Krasnosel'skii, "Equations with nonlinearities of hysteresis type", VII: Int. Konf. Nichtlineare Schwing, Abh. Akad. Wiss. DDR 3 (1977), 437-458.

[21] Y. Mualem, "Modified approach to capillary hysteresis based on a similarity hypothesis", Water Resources Res. 9 (1973), $1324-1331$.

[22] L. Néel, "Théorie des lois d'aimantation de Lord Rayleigh. I - Les déplacements d'une paroi isolée", Cahier Phys. 12 (1942), 1-20. 
[23] L. Néel, "Théorie des lois d'aimantation de Lord Rayleigh. II Multiples domaines et champ coercitif", Cahiers Phys. 13 (1943), $1-20$.

[24] F. Preisach, "über die magnetische Nachwirkung", Z. Phys. 94 (1935), 277-302.

[25] Lord Rayleigh, "Notes on electricity and magnetism. III. On the behaviour of iron and steel under the operation of feeble magnetic forces", Philos. Mag. 23 (1887), 225-245.

[26] A. Visintin, "A model for hysteresis of distributed systems", Ann. Math. Pura Appl. 131 (1982), 203-231.

[21] A. Visintin, "On variable hysteresis operators" (Sonderforschungsbereich 123, Heidelberg, Preprint 204, 1983).

[28] M.P. Weiss, "L'hypothèse du champ moléculaire et la propriété ferromagnétique", J. Phys. (4) 6 (1907), 661-690.

[29] M.P. Weiss and J. de Freudenreich, "Etude de l'aimantation initiale en Jonction de la température", Arch. Sci. Phys. Nat. Geneve 49 $(1916), 5-13$.

Institute for Mathematics, Hochschule der Bundeswehr, Werner-Heisenberg-Weg 39 , 8014 Neubiberg, Munich, Federal Republic of Germany. 